\title{
Sources and Trends of Persistent Organic Pollutants at Three Passive Monitoring Sites in South Africa
}

\author{
Lloyd Shorai Pisa ${ }^{1}$, Nelson Mhlanga ${ }^{2}$, Patricia Mumbengengwi ${ }^{3}$ \\ ${ }^{1}$ Department of Environmental Science and Technology, Marondera University of Agricultural Sciences and Technology, \\ Marondera, Zimbabwe \\ ${ }^{2}$ Oakridge Sustainability Consulting, Milton Keynes, United Kingdom \\ ${ }^{3}$ Department of Travel and Recreation, Chinhoyi University of Technology, Chinhoyi, Zimbabwe \\ Email: 1spisa86@gmail.com
}

How to cite this paper: Pisa, L. S., Mhlanga, N., \& Mumbengengwi, P. (2020). Sources and Trends of Persistent Organic Pollutants at Three Passive Monitoring Sites in South Africa. Journal of Geoscience and Environment Protection, 8, 207-218.

https://doi.org/10.4236/gep.2020.87012

Received: August 13, 2019

Accepted: July 27, 2020

Published: July 30, 2020

Copyright $\odot 2020$ by author(s) and Scientific Research Publishing Inc. This work is licensed under the Creative Commons Attribution International License (CC BY 4.0).

http://creativecommons.org/licenses/by/4.0/

\begin{abstract}
The Stockholm Convention on Persistent Organic Pollutants (SCPOPs) is an international treaty that operates under the patronage of the United Nations (UN) to control certain chemicals that are considered persistent organic pollutants (POPs). They are of great concern because of their adverse effects on human health and the environment. Exposure to POPs may lead to cancer, infertility, respiratory infections, and other health complications. It is therefore critical to study the degree of human and environmental exposure to these POPs. Passive air sampling was conducted in South Africa to evaluate the presence and trends of persistent organic pollutants at Barberspan (rural agricultural), Vanderbijl Park (urban industrial) and Molopo Nature Reserve (isolated nature reserve). Samples were collected in 2012. Passive air samplers using poly were used for sampling. The Buchi System B-811 automatic extractor was used to analyze for dichloro-diphenyl-trichlorethane (DDT), pentachlorobenzene (PeCB), hexechlorobenzene ( $\mathrm{HCB})$ polychlorinated biphenyls (PCBs) and hexachlorocyclohexane (HCH). Sampling was conducted for a period of one year. POPs concentrations were generally low at all sites, but Vanderbijl Park had the highest concentrations. Seasonal changes in concentrations were much the same at the three sites with temperatures and wind patterns being major factors. The presence of these chemicals in Molopo Nature Reserve and Barberspan is evidence of long range transportation over dry semi desert areas. Back-trajectories indicate possible sources, including areas where DDT is used for malaria control. The presence of POPs is areas they have never been in use poses health and environmental risks in these areas.
\end{abstract}




\section{Keywords}

Persistent Organic Pollutants, Passive Air Sampling, Long Range

Transportation

\section{Introduction}

Persistent Organic Pollutants (POPs) are of anthropogenic origin and a major concern because of their toxicity, persistence, long range transportation, and potential to bio-accumulate. POPs are resistant to photolytic, biological, and chemical degradation. Their physical and chemical properties enable the compounds to undergo long range transportation, allowing the pollutants to become widely distributed geographically through air (they are semi-volatile), water and biota, even to regions where they have never been used or produced (Ritter et al., 2005). Because of the lipophilic nature, these pollutants tend to accumulate in matrices rich in organic matter such as soil, sediment and biota, and can bio-accumulate in food web (Schecter et al., 2006). Human exposure to POPs can lead to health problems such as dermal effects, liver, kidney illnesses, defects of the immune, reproductive, nervous, and endocrine systems, and even cancer.

The Stockholm convention on Persistent Organic Pollutants to protect human health and the environment from POPs through internationally agreed policies and actions aimed at reducing and eliminating their release into the environment became international law on 17 May 2004. The convention bans and/or restricts the production and use of the intentionally produced POPs and it aims at reducing releases of unintentionally produced POPs, which are formed as byproducts of combustion and industrial processes (UNEP, 2005). The convention has successfully leaded to the ban of most POPs. The convention supports the replacement of harmful POPs with safer, cost-effective alternatives. However, this process may pose a challenge to developing countries, as they lack the financial and technological recourses to use and manufacture less harmful chemicals and develop new techniques. The convention calls on developed nations to share their knowledge and lend financial support to developing countries and economies in transition by aiding their transition to more suitable alternatives (UNEP, 2001). The Stockholm Convention, of which South Africa is a party, carries a number of obligations and expectations. Based on the obligation to develop a National Implementation Plan (NIP), the State is obliged to reduce or terminate all sources of POPs within the Stockholm Convention's provisions, provided that timely and sufficient means have been made available. This therefore implies that the state should know the environmental levels of these POPs whereby priority sources and hotspots can be targeted for interventions. Since much of this information is either old or lacking, research needs to be undertaken (Ross et al., 2004). In some countries, due to lack of alternative compounds for industrial 
use and financial constrains, some POPs compounds like dichloro-diphenyltrichlorethane (DDT) are still in use. For example South Africa still uses DDT for malaria control (Bouwman \& Kylin, 2009).

Polychlorinated Biphenyls (PCBs) and hexachlorocycohexane ( $\mathrm{HCH}$ ) are commonly found in most air samples. PCBs are used in electrical transformer hydraulic fluids and fire retardants. Other sources of POPs are accidental spillages and vitalization from old stock piles. PCBs can also be unintentionally produced as byproducts of industrial processes. $\mathrm{HCH}$ is used as an insecticide to control insects of cotton, stem borers and wire-worms in cotton. Other sources include old stock piles and leaching from waste disposal sites.

Other studies have shown that POPs can be found in areas in which they were not in use. Under conditions of climatic variation, atmospheric circulation patterns may play a role in POPs movement and distribution. This shows that not only are the industrial areas affected by the use of POPs but rather a greater area. Long range transportation can be a major source of these pesticides (Ritter et al., 2005; Wania \& Mackay, 1996).

Most POPs are used as pesticides or herbicides in agriculture. POPs such as DDT and $\mathrm{HCH}$ are used to control a variety of agricultural and horticultural pests. DDT is also used for malaria control in the Limpopo province (Batterman et al., 2008; WHO, 2008). It is mainly used because of its effectiveness and low cost. However is does have consequences. Due to long range transportation the chemical can spread over a large area to regions it is not known to be in use.

In 2008, a Monitoring Network-Africa project based on the Memorandum of the United Nations Environmental Program (UNEP) was been carried out throughout Africa. South Africa provided three sampling sites, a rural agricultural site, remote isolated site and one industrial site. Molopo Nature Reserve was the background site, Barberspan Bird Sanctuary the rural agricultural site, and Vanderbijl Park was an industrial site. Molopo Nature Reserve (25.80229S, 2296975E) has no industrial activity and no potential sources of POPs are evident. Barberspan (26.67200S, 25.567930E) also has no known use of POPs within this area. Vanderbilj Park (26.70665S, 27.85857E) on the other hand is an industrial site. The major activities in the area include an iron and steel manufacturing plant. There is also a petrochemical plant, power plant and coal mine. The site contains a number of potential sources of POPs.

This study looks at the trends of DDT, pentachlorobenzene (PeCB), hexachlorobenzene (HCB), $\mathrm{HCH}$, and PCBs in air at the three sites. The study will help understanding of POPs movement, distribution and ability to undergo long range transportation.

\section{Methods}

\subsection{Study Site and Sampling}

Figure 1 shows three sample sites where passive air samplers were placed. Three sampling sites were selected based on their land uses and location. Vanderbijlpark 


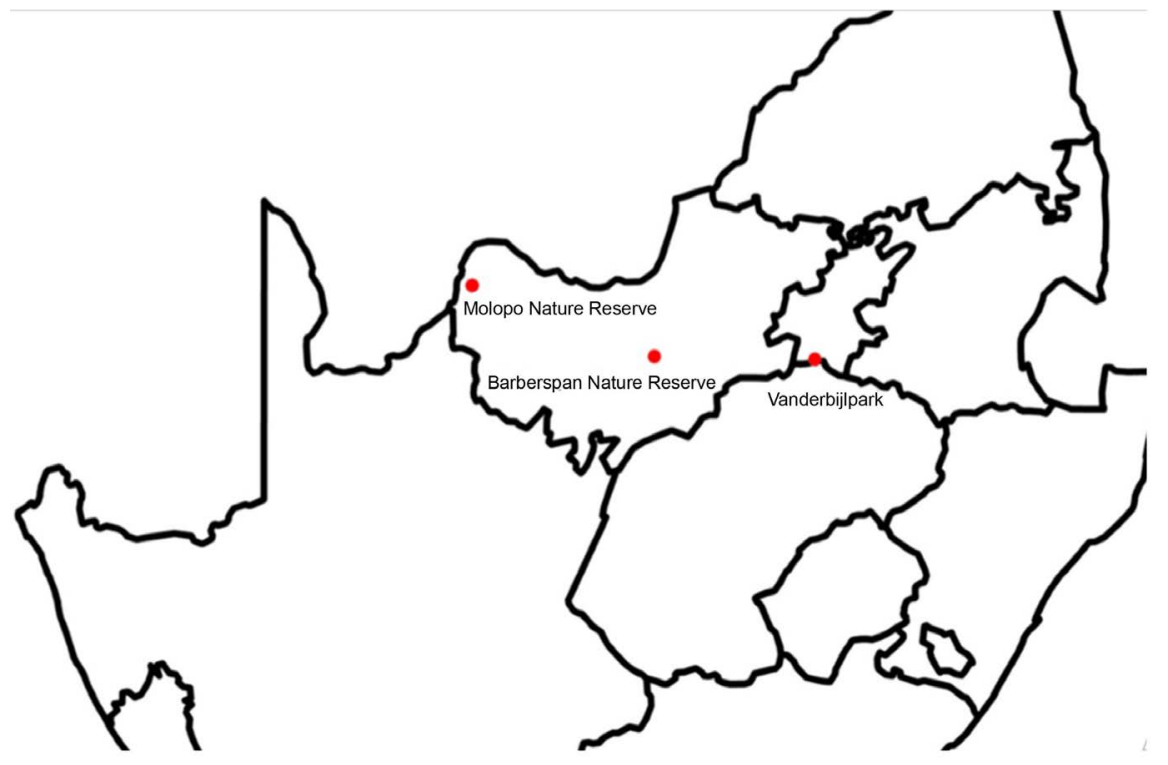

Figure 1. Study sites where passive are samples were collected (Molopo Nature Reserve, Barberspan Nature Reserve and Vanderbiljpark).

(26.7S, 27.8E) is highly industrial with a population of about 220,000. It lies next to the Vaal River and is in the south of the Gauteng Province. The major activities in the area include an iron and steel manufacturing plant, petrochemical plant, power plant, and a coal mine. A major steel company, Vanderbijlpark Steel is located in the town. The town is also surrounded by other industrial towns such as Vereeniging and Sasolburg. Barberspan Nature Reserve (26.6S, 25.5E) was the second site. It is located in the North West Province, $17 \mathrm{~km}$ north-east of Delareyville at an altitude of between $1345 \mathrm{~m}$ and $1360 \mathrm{~m}$ above mean sea level. The sanctuary has a 3118 ha wetland. The vegetation is mainly flat grassy plains and a few scattered trees. Molopo Nature Reserve (25.8S, 22.9E) was chosen as a remote background site. The reserve is located on 24,000 ha of conserved land. It lies on the Botswana border in the far west of the North West Province, $250 \mathrm{~km}$ north of Vryburg, and $7 \mathrm{~km}$ west of the village of Vostershoop. The vegetation is Kalahari grassland. The area is arid and receives very little rain. A large number of bird species and wildlife are found at the reserve, including eland, blue wildebeest, gemsbok, red hartebeest, kudu, zebra impala waterbuck, and springbok. Air samples were collected using passive air sampling (REF). The passive air sampling device had two stainless steel bowls attached to the common axes and acted as a protective chamber for the polyurethane foam (PUF) filter. Before deployment, PUF filters, $(15 \mathrm{~cm}$ diameter, $1.5 \mathrm{~cm}$ thick, $0.030 \mathrm{den}$ sities, type N3038, GumotexBreclav, (Czech Republic) were cleaned for eight hours with acetone and further eight hours with dichloromethane. The filters were wrapped in two layers of aluminum foil, placed into zip-lock polyethylene bags, and kept in the freezer prior to deployment. The chambers were washed and rinsed with acetone before installation. The polyurethane foam filters were exposed to the air for four weeks. On collection, the exposed filters were 
wrapped in two aluminum foils, labeled and kept at a temperature of 5 during transportation. For storage the samples were stored at -18 before analysis. Samples were collected over a period of one year at monthly intervals from January 2008 to December 2008, and shipped to RECETOX, Czech Republic for analyses.

\subsection{Analysis}

Standards were purchased from wellington Laboratories in Canada. These included calibration sets, natives, and mass labeled. Automated warm Soxhlet extraction (60 minutes Soxhlet followed by 30 minutes of solvent rinsing) with toluene in B-811 extraction unit (Buchi, Switzerland) was used for extraction. Samples are spiked before extraction. With ${ }^{13} \mathrm{C}$ PCDDs/Fs according to EN-1948), ${ }^{13} \mathrm{C}$ dl-PCBs $(77,81,126,169,105,114,118,123,156,157,167,189)$ and ${ }^{13} \mathrm{C}$ BDEs $(28,47,66,100,99,85,154,153,183,209)$ the concentrated extraction were cleaned-up on $\mathrm{H}_{2} \mathrm{SO}_{4}$ modified ( $30 \% \mathrm{w} / \mathrm{w}$ ) silica column, eluted with $40 \mathrm{ml}$ $\mathrm{DCM} / n$-hexane mixture $(1: 1)$. Fractionation was achieved in micro column (6 $\mathrm{mm}$ id) containing from bottom to top: $50 \mathrm{mg}$ silica, $70 \mathrm{mg}$ charcoal/silica (1:40) and $50 \mathrm{mg}$ of silica. $5 \mathrm{ml}$ of toluene and $5 \mathrm{ml}$ of DCM/cyclohexane mixture (30\%) were used to prewash the column. The sample was applied and eluted with $9 \mathrm{ml}$ $\mathrm{DCM} /$ cyclohexane mixture (30\%) in fraction 1 (PBDEs, mono-ortho dl-PCBs) and $40 \mathrm{ml}$ of toluene in fraction 2 (PCDDs/Fs, non-ortho dl-PCBs). Each fraction was concentrated using stream of nitrogen in a TurboVap II (Calliper Life Sciences, USA) concentrator unit and transferred into an insert in a vial. The syringe standards $\left({ }^{13} \mathrm{C}\right.$ PCDDs, ${ }^{13} \mathrm{C}$ PCBs $70,111,138$ and $170,{ }^{13} \mathrm{C}$ BDEs 77 and 138) were added to all samples, the final volume was $50 \mathrm{uL}$.

The following compounds were analysed. HRGC/HRMS instrumental analysis (PCDDs/Fs, dl-PCBs) was performed on a 7890A GC (Agilent J\&W) equipped with a $60 \mathrm{~m} \times 0.25 \mathrm{~mm} \times 0.25 \mu \mathrm{m}$ DB5-MS column (Agilent J\&W, USA) coupled to an Auto Spec Premier MS (Waters, Micro mass, UK). The MS was operated in EI+ mode at the resolution of $>10,000$. BDEs were analysed on the same system using $15 \mathrm{~m} \times 0.25 \mathrm{~mm} \times 0.10 \mu \mathrm{m}$ DB5 column (Agilent J\&W). For BDE 209, the MS resolution was set to $>5000$.

\subsection{Quality Assurance, Quality Control}

Spiking with the surrogate standards prior to extraction was used to determine recoveries. For PCDDs/Fs recoveries of isotopically labeled standards were 55\% $90 \%$ and $60 \%$ and $90 \%$ for dl-PCBs. BDEs recoveries ranged between $60 \%$ and $110 \%$. The data was correctly recovered. All the compounds had laboratory blanks under quantization. Field blanks consisted of pre extracted PUF disks and were taken on each sample site. These were extracted and analyzed in the same manner as the field blanks.

\subsection{Generation of Air Mass Back Trajectories}

Daily air mass trajectories at $200 \mathrm{~m}$ were generated using the HYSPLIT model of 
the American National Oceanic and Atmospheric Administration, NOAA. Global Data Assimilation System (GDAS) achieve meteorological data was used. The trajectories were overlaid to give maps for each month.

\section{Results}

Figures 2-6 shows the monthly $\mathrm{HCH}, \mathrm{PCB}$, DDT, $\mathrm{HCB}$, and $\mathrm{PeCB}$ concentrations at the three sites. They show the trends of POP concentrations in all three areas throughout the year. The concentrations of industrial chemicals, $(\mathrm{PeCB}$, $\mathrm{PCB}$, and $\mathrm{HCB}$ ), were generally lower at all three sites. The $\mathrm{HCH}$ concentrations (Figure 2) increase in July at all three sites. Vanderbijl Park air showed a sharp decrease in concentrations between September and October. PeCB concentrations, Figure 3, were very low at all three sites, ranging between $0-2.3 \mathrm{ng} /$ filter. The remote site, Molopo however had traces of the POP. The trend is generally

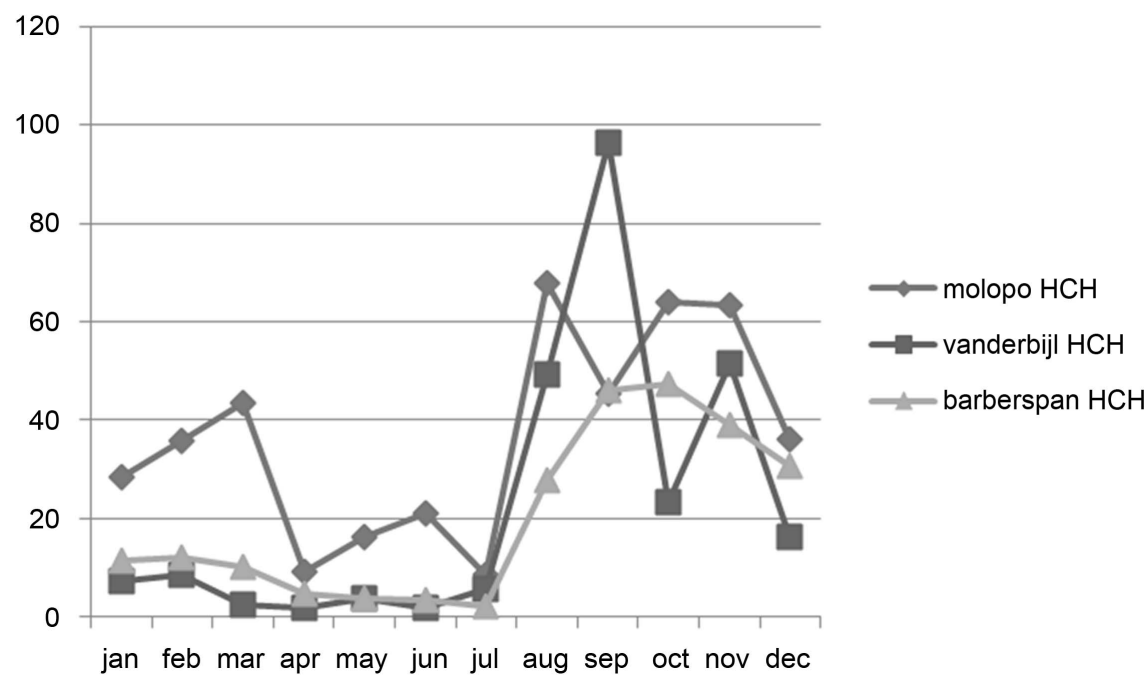

Figure 2. Sum of HCH concentrations on the three sites in ng/filter.

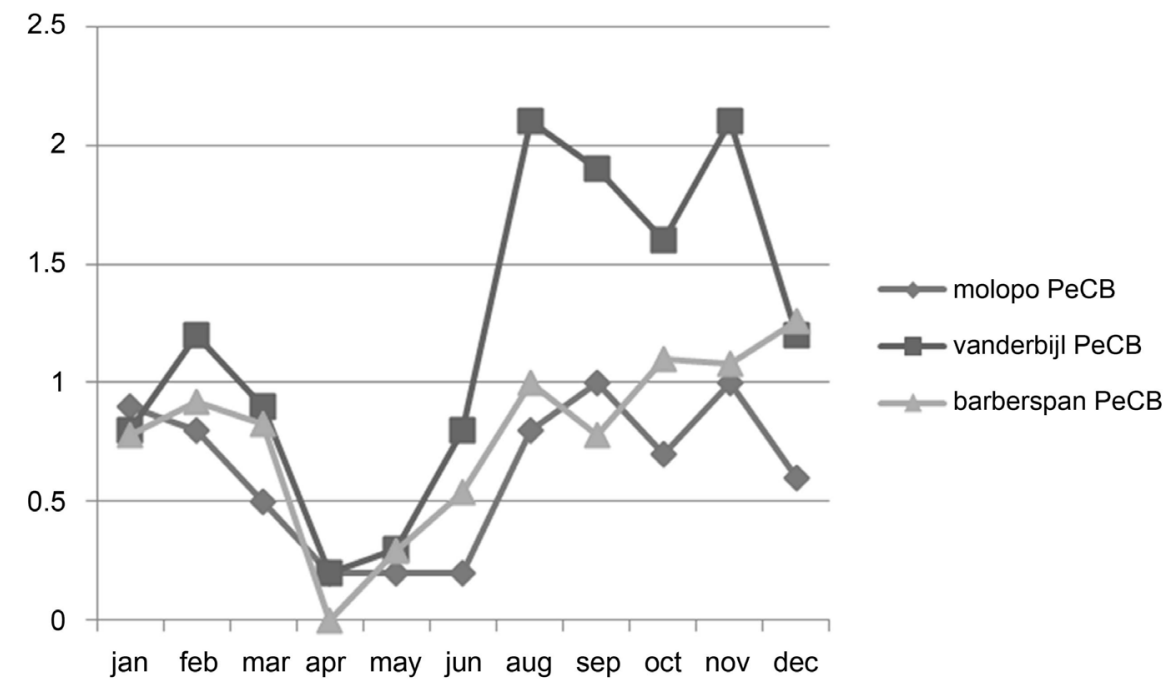

Figure 3. Sum of PeCB concentrations on the three sites in ng/filter. 


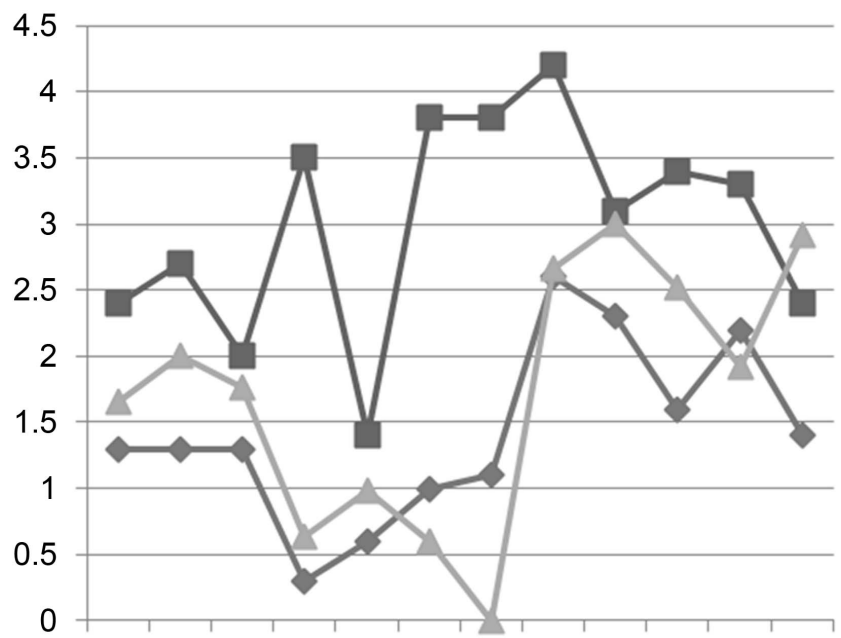

$\sim$ molopo HCB

- vanderbijl HCB

$\simeq$ barberspan $\mathrm{HCB}$

Figure 4. Sum of HCB concentrations on the three sites in ng/filter.

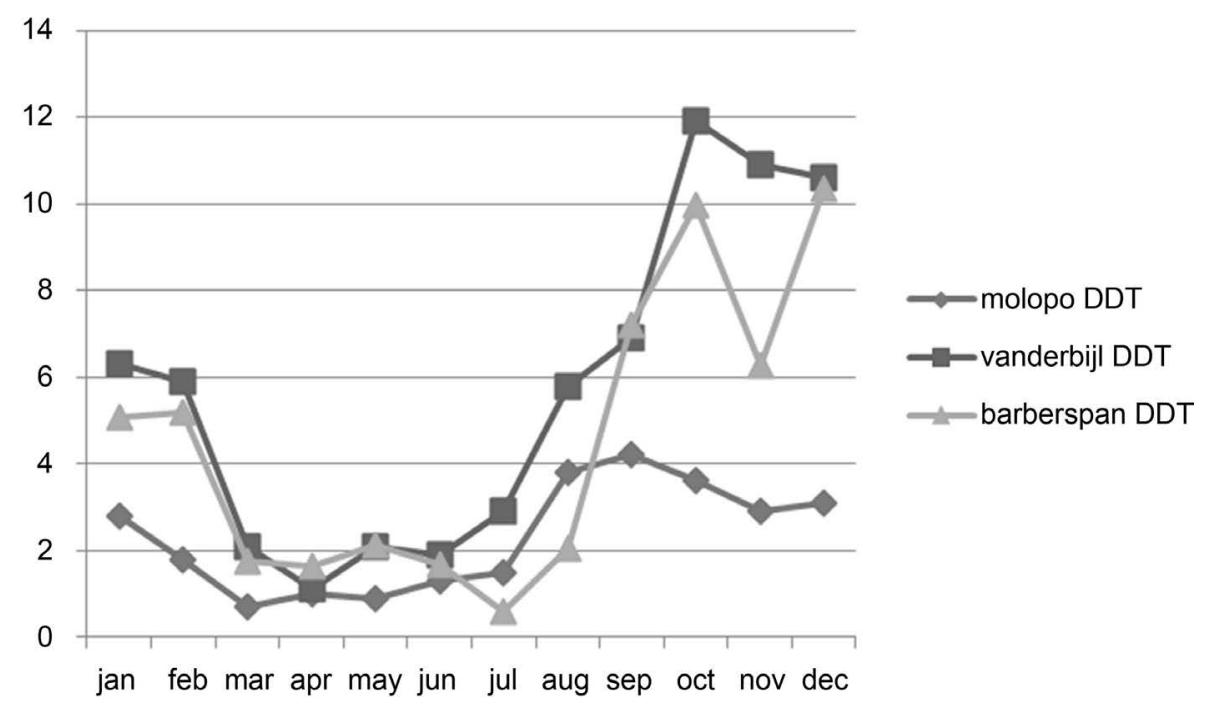

Figure 5. Sum of DDT concentrations on the three sites in ng/filter.

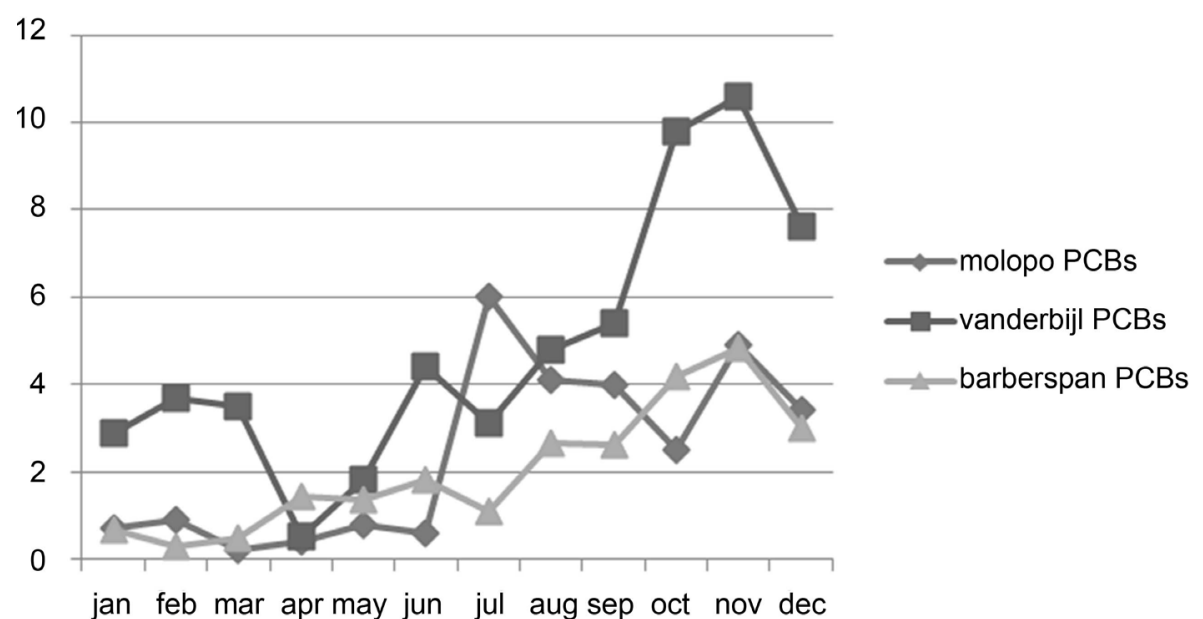

Figure 6. Sum of PCBs concentrations on the three sites in ng/filter. 
an increase in concentration as the year progresses. HCB, Figure 4, also had a low concentration ranging from $0-4.2 \mathrm{ng}$ /filter. Barberspan recorded to lowest concentration of $0 \mathrm{ng} /$ filter in July. Traces of the chemical were also found in the isolated area Molopo. DDT, Figure 5, was evident on all three sites. Trends were fairly similar on all three sites, with a general dip in concentration between March and June. Concentrations of DDT again shot up in July till year end. PCB, Figure 6, concentrations were lowest in Barberspan and Molopo. Vanderbijl recorded the highest concentration. The concentrations increased as the year progressed on all three sites.

\section{Back Trajectory Maps}

The above maps show backward trajectories from Molopo sampling site. Each map shows air mass movements for each month. Each trajectory shows air movement for a period of 24 hours before it reached the sampling area (Figure 7).

The above maps show backward trajectories from Barberspan Bird Sanctuary sampling site. Each map shows air mass movements for each month. Each trajectory shows air movement for a period of 24 hours before it reached the sampling area (Figure 8).

The above maps show backward trajectories from Barberspan Bird Sanctuary sampling site. Each map shows air mass movements for each month. Each trajectory shows air movement for a period of 24 hours before it reached the sampling area (Figure 9).
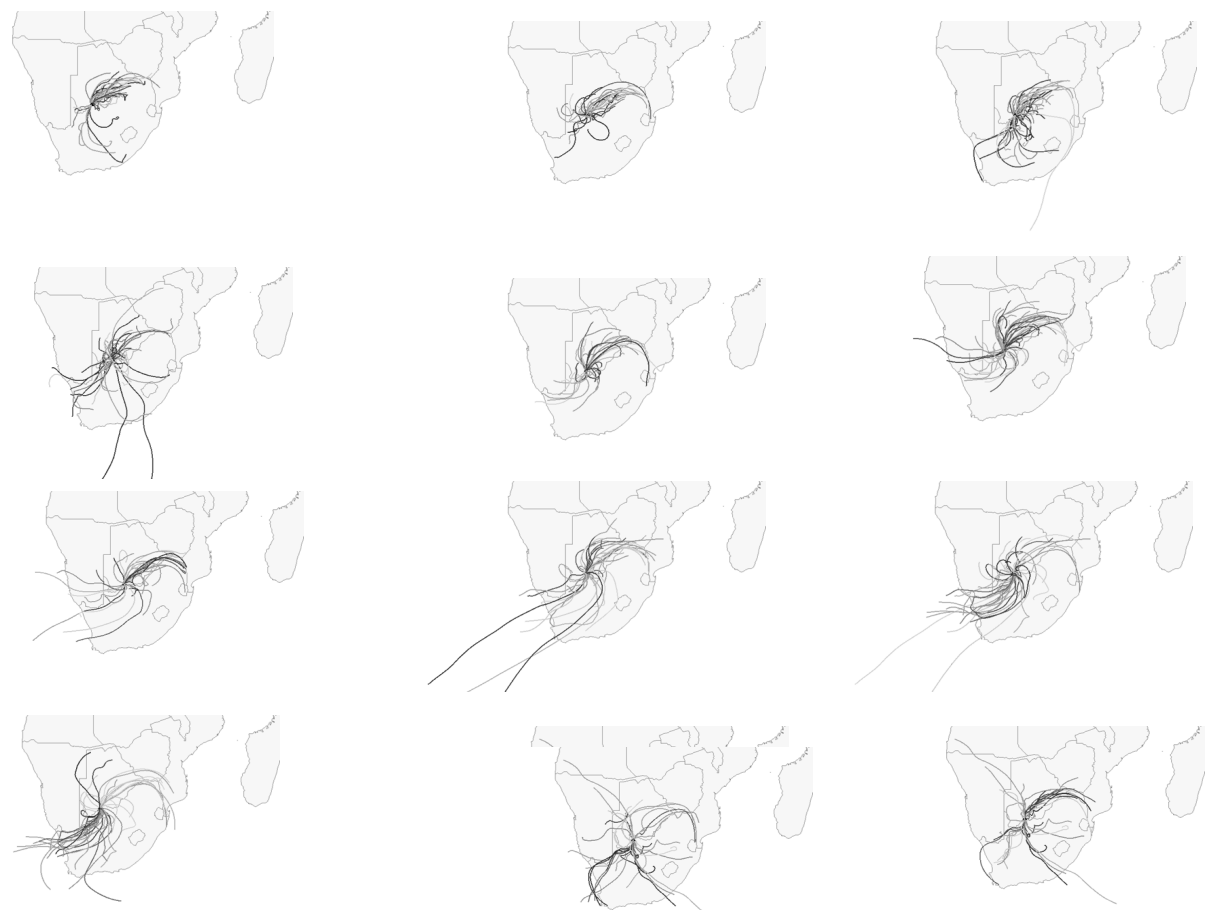

Figure 7. Back trajectory maps for Molopo Nature Reserve for 12 sampling periods in 2012. 


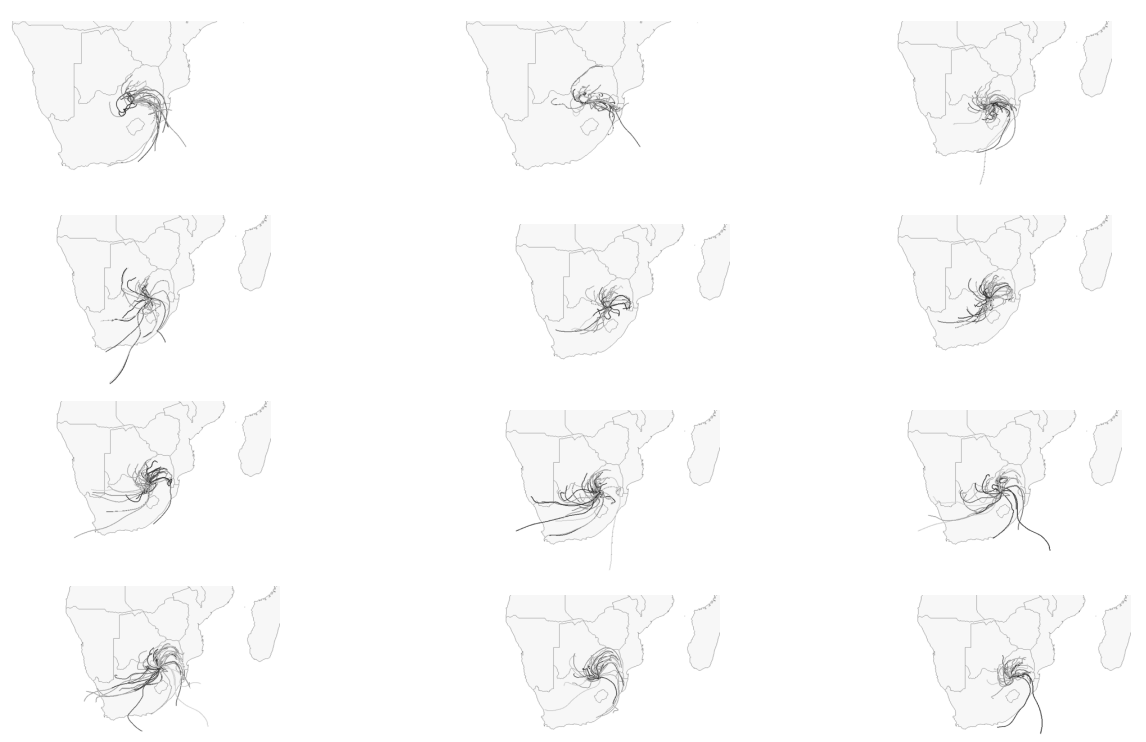

Figure 8. Monthly back-trajectory maps for Barberspan Bird Sanctuary in 2012.
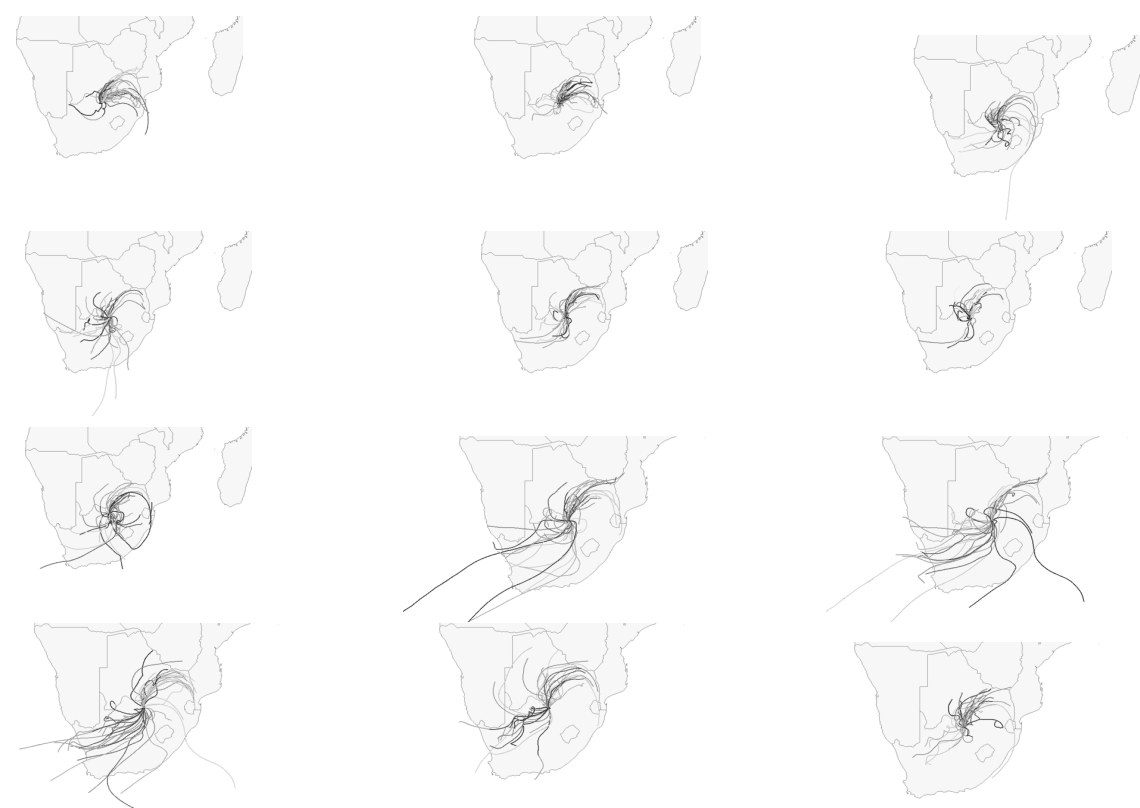

Figure 9. Back trajectory maps for Vanderbljpark for 12 sampling periods in 2012.

\section{Discussion}

\section{$\mathrm{HCH}$}

$\mathrm{HCH}$ concentrations rose around July on all three sites (Figure 2). This could be attributed to $\mathrm{HCH}$ being volatile. $\mathrm{HCH}$ still remains in the environment due to its extensive historic use, present use of lindane (for the control of lice and mites) in several countries, unused stockpiles from earlier manufacturing, as well as leachates from waste disposal sites (Bhatt et al., 2009; ATSDR, 2007). According to Sturt Harrad (2008), a $\mathrm{HCH}$, was used for pest control in the past and South Africa had the largest use in Africa. Historical use may also be responsible 
for the presence of the $\mathrm{HCH}$ in air. Barbenspan is located in an agricultural community. The use of $\mathrm{HCH}$ in surrounding area for insect control can also have contributed to their high concentration in these areas. Strong winds and wind patterns may have resulted in long range transportation of the pollutant to Molopo Nature Reserve and Barbenspan Bird Sanctuary. Vanderbijl Park also had a rise in concentration towards year end. The rise in yearend are likely to be due to temperature rises in the summer and hence an increase in volatilization. The period between July and September gave the highest concentrations of $\mathrm{HCH}$ on all three sites (figure). Heavy winds could be a potential cause to the sharp rise. The season between July and October is relatively dry and South Africa experiences an increase in veld fires. This is mostly pronounced in the Limpopo Province. Back trajectories indicate air mass movements from that region to the three sampling sites. These could also be potential sources of HCH. A deep in concentration was observed between March and June. This is likely to be due to deposition and low wind movement. A drop in temperature and rainfall could have given rise to the decrease.

PCBs are an industrial chemical. PCBs were introduced into the environment from extensive use in industrial applications due to their chemical inertness, low flammability, and electrical insulating properties. Concentrations were generally low in all sampling sites (Figure 6). Vanderbijl Park recorded the highest concentrations likely because of the industrial activities in the area. Though concentrations were low in the other two sites, Barberspan Bird Sanctuary and Molopo Nature Reserve, it however reveals the presence of long range transportation. According to Aries et al., 2004, PCBs are also produced by various thermal processes in the iron and steel industries. This may be the source of PCBs in Vanderbijl Park. An increase is also noted in summer most likely due to high temperatures and winds which enhance volatilities. The presence of PCBs at Barberspan Bird Sanctuary is also a point of concern as PCBs may lead to reduced reproductive potential, developmental and toxic effects such as malformation, pericardial oedema, liver lesions, and lethality to embryos leading to population decreases (Barron et al., 1995).

PeCB concentrations were the lowest for all POPs investigated. Vanderbijl Park did however record the highest concentration. Concentrations reduced between January and April on all three sites. This may be due to deposition due. A rise was again noted as the windy season began and temperatures picked up. All sites had a missing result for July. The presence of the compound at remote sites like Molopo Nature Reserve is likely due to long range transportation. Back trajectories in Figure 7, on the background sites show that some air masses passing through the sites go over a number of industrial areas such as Gaborone, Rustenburg and Johannesburg. This indicates POPs can travel long distances and hence there impact on the environment stretches far beyond their area of use. Mining and agricultural activities are the likely sources of POPs.

DDT was detected on all three sites and a similar pattern was noted as shown in Figures 7-9. DDT level increased from around August till year end, with the 
sharpest rise in Barberspan Bird Sanctuary. Concentrations were also high at the beginning of the year. DDT is sprayed in the Limpopo Province for malaria control. Indoor Residual Spraying is done towards the end of the year. The high concentrations at the beginning of the year are likely to be excess from the DDT sprayed the years before, that is 2007 and before. The concentrations in air reduced in February to July. The reduction could be due to deposition and rainfall. A drop in wind speeds and temperature are potential causes as well. Higher concentrations can be detected around August. Back trajectories show that air masses from the Limpopo region flow over the three sites. This is the likely source of DDT. Long range transportation contributes greatly to DDT distribution throughout South Africa. The increase in winds in August aids in distribution and movement of the chemical. Volatilities from soil are also possible due to increases in temperatures.

$\mathrm{HCB}$ is both an industrial and agricultural chemical. According to Barber et al., 2005, HCB was used as a fungicide in the 1960s. HCB can also be formed as a by-product of combustion processes and solvent production. Figure 9 shows that residents in the Gautang Province and areas near Vanderbijl Park are at risk. The sources of HCB in Vanderbijl Park could be the mines or petrol manufacturing industry. The background sites also had traces of HCB but concentrations were low.

\section{Conclusion}

Passive air sampling of the three sites in South Africa indicates that POPs are present in South African air. Though concentrations are generally low, there is need for constant monitoring and investigations on the fate of POPs. The potential harm to sensitive areas in unknown and hence precaution should be taken to avoid damage. The presence of these POPs in Molopo Nature Reserve and Barberspan Bird Sanctuary is also of concern. The ability of POPs to move or travel to other areas increases the environmental impact of the chemicals spatially. There is need to determine a ceiling for the "acceptable" levels of POPs within the atmosphere so as to develop proper monitoring systems and regulations to industries responsible for the emissions. Public awareness is also important as people have the right to know what they are being exposed to and its possible implications on them and their environment.

\section{Conflicts of Interest}

The authors declare no conflicts of interest regarding the publication of this paper.

\section{References}

ATSDR (2007). Agency for Toxic Substances and Disease Registry. Hexachlorocyclohexane: Toxicological Profile.

Barron, M. G., Galbraith, H., \& Beltman, D. (1995). Comparative Reproductive and De- 
velopmental Toxicology of PCBs in Birds. Comparative Biochemistry and Physiology, 112C, 1-14. https://doi.org/10.1016/0742-8413(95)00074-7

Batterman, S. A., Chernyak, S. M., Gounden, Y., Matooane, M., \& Naidoo, R. (2008). Organochlorine Pesticides in Ambient Air in Durban, South Africa. Science of the Total Environment, 39, 119-130. https://doi.org/10.1016/j.scitotenv.2008.02.033

Bhatt, P., Kumar, M. S., \& Chakrabarti, T. (2009). Fate and Degradation of POP-Hexachlorocyclohexane. Critical Reviews in Environmental Science \& Technology, 39, 655-695. https://doi.org/10.1080/10643380701798306

Bouwman, H., \& Kylin, H. (2009). Malaria Control Insecticide Residues in Breast Milk: The Need to Consider Infant Health Risks. Environmental Health Perspectives, 117, 1477-1480.

Ritter, L., Solomon, K. R., \& Forget, J. (2005). Persistent Organic Pollutants: An Assessment Report on DDT, Aldrin, Dieldrin, Endrin, Chlordane, Heptachlor, Hexachlorobenzene, Mirex, Toxaphene, PCBs, Dioxins, and Furans. Report for the International Programme on Chemical Safety (IPCS) within the Framework of the Inter-Organization Programme for the Sound Management of Chemicals (IOMC, $44 \mathrm{p}$.

Ross, E. (2004). Yushchenko's Dioxin Poison Level More Than 6000 Times Higher than Normal. http://www.nctimes.com

Schecter, A., Birnbaum, L., Ryan, J. J., \& Constable, J. D. (2006) Dioxins: An Overview. Environmental Research, 101, 419-428. https://doi.org/10.1016/j.envres.2005.12.003

UNEP (2001). United Nations Environment Programme. Stockholm Convention on Persistent Organic Pollutants. Geneva, Switzerland.

UNEP (2005). United Nations Environment Programme. Ridding the World of POPs: A Guide to the Stockholm Convention on Persistent Organic Pollutants. Geneva, Switzerland.

Wania, F., \& Mackay, D. (1996). Tracking the Distribution of Persistent Organic Pollutants. Environmental Science \& Technology, 30, 390-396.

https://doi.org/10.1021/es962399q

WHO (2008). World Malaria Report 2008. Geneva: World Health Organization. 